\title{
Analogous of Wien's law for the optimal wavelengths selection in bi-spectral method used for temperature measurement of surfaces exhibiting non-uniform emissivity, and general methodology for the multi-spectral method
}

\author{
By C. RODIET ${ }^{\star}$, B. REMY*, A. DEGIOVANNI
}

\author{
* LEMTA-ENSEM, CNRS - F-54504 Nancy University, France, christophe.rodiet@univ-lorraine.fr
}

\begin{abstract}
In this paper, a law similar to that of the Wien's law, but to determine the optimal wavelengths in a mono-spectral and a bi-spectral methods (without the Wien's approximation, i.e. for a Planck law) used for temperature measurement of surfaces exhibiting non-uniform emissivity, and a more general methodology (based on the ordinary least squares method) to obtain the optimal wavelengths selection in a multi-spectral method is presented. The goal consists of minimizing the standard deviation of the estimated temperature (optimal design experiment). For the multi-spectral method, two cases will be treated: optimal global, and optimal constrained (to the spectral range of the detector, for example) wavelengths selection are presented. The estimated temperature results obtained by different models taking into account a secondorder polynomial transfer function and including the emissivity variations and for different number of parameters and wavelengths are compared. Different selection criteria are presented. These points are treated from theoretical, numerical and experimental points of view.
\end{abstract}

\section{Nomenclature}

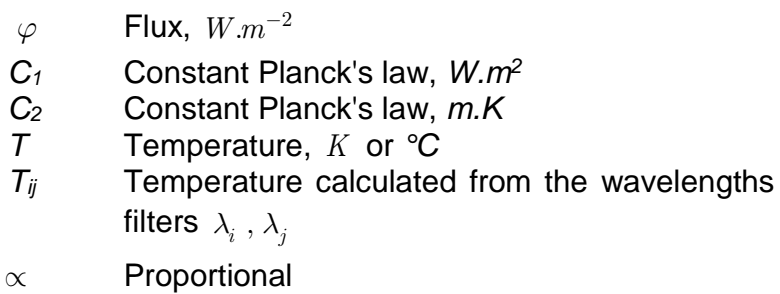

\begin{tabular}{|c|c|}
\hline & 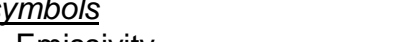 \\
\hline$\varepsilon$ & Emissivity \\
\hline$\lambda$ & Wavelength, $m$ \\
\hline$\chi$ & Sensitivity \\
\hline Indi & exponents and other symbols \\
\hline$\lambda$ & Spectral, or wavelength \\
\hline$m$ & Mean \\
\hline$i, j, k$ & Number of filter \\
\hline
\end{tabular}

\section{Introduction}

These last years in parallel with industrial development, optical measurement methods were increasingly used for measuring space and/or time distributions of temperature in extreme conditions (such as Tokamaks) or for the thermal characterization of weakly reflective opaque materials at high temperatures [1]. These methods presents the advantage of being weakly intrusive and allow performing remote measurement, which are usually done by infrared cameras, quantum detectors, or photomultipliers in the case of measurements at shorter wavelengths [2]. The measurement difficulties are numerous, such as taking into account the reflection on the sample, or spatial and temporal variations of the emissivity of the material, making it non-uniform over the sample surface, especially at high temperature where significant oxidation phenomena can occur. One solution is to make a measurement by the multi-spectral method [2-9], of which a state-of-theart has been made by $[4,8]$. Even if the idea is interesting, its implementation is tricky because of the difficulty to choose the adapted wavelengths $\lambda_{i}$. Indeed, they must be chosen "close enough" to overcome emissivity variations of the material, but not "too close" to obtain an uncertainty on the measured temperature lowest as possible [9].

After a presentation of analogous Wien's laws for "optimal" wavelengths selection for mono-spectral and bispectral measurements, the theoretical principle of the multi-spectral methods is presented. Next, several models are validated numerically through Monte-Carlo simulations for different spectral emissivity variations, and compared experimentally. The facility is presented in section 6 , and the considered variations of emissivity used to validate the theoretical models for estimating temperature through an inverse technique based on an ordinary least squares method are shown in section 5. Two different cost functions $((8)$ and (10)) will be used to estimate this temperature by inverse method: the first uses fluxes ratio and the Wien approximation (7), and the second uses the fluxes (9) (Planck's law without any fluxes ratio).

\section{Analogous Wien's law for optimal wavelengths selection : mono-spectral method}

Calling $f(\lambda)$ the global spectral transfer function including all unknowns (emissivity $\varepsilon(\lambda)$, sample area, quantum efficiency...), the flux emitted by an object is defined by the Planck's law (1) which can take a simpler form (named Wien's approximation) if $\lambda T<<C_{2} \approx 14400 \mu m . K$ : 


$$
\varphi(\lambda, T)=f(\lambda) M^{o}(\lambda, T)=f(\lambda) \frac{C_{1} \lambda^{-5}}{\exp \left(\frac{C_{2}}{\lambda T}\right)-1} \underset{\begin{array}{c}
\text { Wien's } \\
\text { approximation }
\end{array}}{\simeq} f(\lambda) C_{1} \lambda^{-5} \exp \left(\frac{-C_{2}}{\lambda T}\right), \quad \forall \lambda T<<C_{2} \approx 14400 \mu m . K
$$

Considering $\lambda$ as a parameter, by differentiating the Planck's law (1) with respect to temperature and equating the differential terms to errors, it can be shown that the relative error on the temperature is:

$$
\frac{e_{T}}{T}=\frac{e_{\varphi}}{\varphi} \frac{\lambda T}{C_{2}}\left(1-\exp \left(\frac{-C_{2}}{\lambda T}\right)\right) \underset{\substack{\text { Wien's } \\ \text { approximation }}}{\simeq} \frac{e_{\varphi}}{\varphi} \frac{\lambda T}{C_{2}}
$$

Furthermore, if it is assumed that $e_{\varphi} / f(\lambda)=k$ (with $k$ a constant), find the wavelength that minimizes (2) under the Wien's approximation, leads to:

$$
\min _{\lambda}\left(\frac{e_{T}}{T}\right) \Rightarrow \frac{\partial}{\partial \lambda}\left(\frac{e_{T}}{T}\right)=0 \Rightarrow \frac{\partial}{\partial \lambda}\left(\frac{k T}{C_{2}} \frac{\lambda}{M^{o}}\right)=0 \Leftrightarrow \lambda_{o p t}^{\text {Wien }} T=\frac{C_{2}}{6} \approx 2400 \mu m K=C_{M W}^{R}
$$

Accordingly, (3) shows that under the Wien's approximation and for the mono-spectral measurements, the wavelength that minimizes the relative error on the temperature is defined from an analogous to Wien's law: $\lambda_{o p t}^{\text {Wien }} T=C_{M W}^{R} \approx 2400 \mu m K$. Note that, equating the errors to the standard deviation (to the least squares sense), minimizing (2) is equivalent to minimize the standard deviation $\sigma_{T}$ of the temperature for a constant standard deviation $\sigma_{\varphi}$ of the flux (cf. section 4).

It seems legitimate to wish extend this law, but for a flux defined by Planck's law. The equation to solve being nonlinear (cf. (2) and (3)), the method consists in search numerically for several temperatures $T_{i}$ the wavelengths $\lambda_{i}^{\text {opt }}$ that minimize $e_{T_{i}} / T_{i}$ (cf. figure 1), then to determine the constant $C_{M P}^{R}=\lambda_{\text {opt }}^{\text {Planck }} T$ by a least squares method, such as:

$$
\hat{C}_{M P}^{R}=\arg \min _{C_{M P}^{R}} \sum_{i}\left(\lambda_{i}^{\text {opt }}-\frac{C_{M P}^{R}}{T_{i}}\right)^{2} \Rightarrow \lambda_{\text {opt }}^{\text {Planck }} T=\hat{C}_{M P}^{R} \approx 2410.3 \mu m K
$$

The residues defined by $r\left(T_{i}\right)=\lambda_{i}^{\text {opt }}-2410.3 / T_{i}$ are plotted in figure 2. Low residue values (cf. figure 2) corroborates a good fit of the law $\lambda_{\text {opt }}^{\text {Planck }} T=\hat{C}_{M P}^{R} \approx 2410.3 \mu m K$ to find the optimal wavelengths for mono-spectral measurements (with Planck's law).

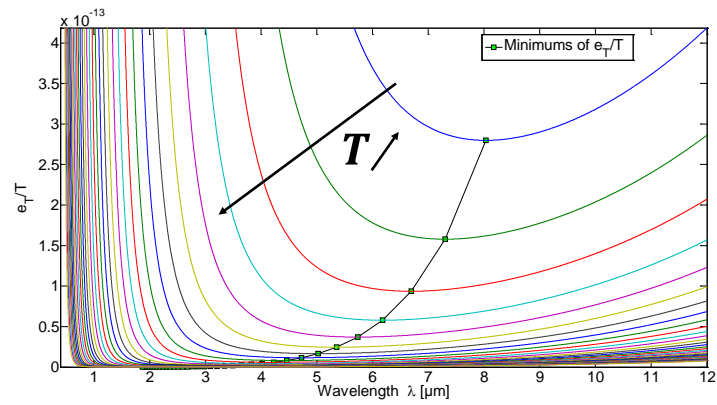

Fig. 1. Minimums of $e_{T} / T \propto \lambda / M^{o}$ for $T \in[300 K ; 1300 K]$ (1 curve out of 30$)$.

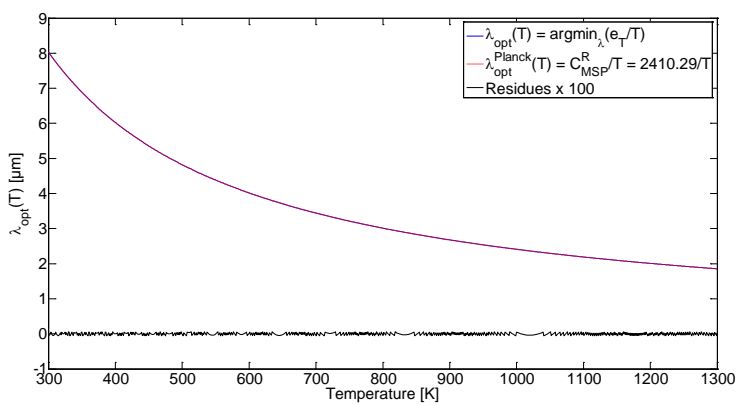

Fig. 2. Optimal wavelengths obtained numerically and by the law $\lambda_{\text {opt }}^{\text {Planck }}=\hat{C}_{M P}^{R} / T$ for $T \in[300 K ; 1300 K]$. The maximum residue is of about $5.10^{-4} \mu \mathrm{m}$.

Note that, minimizing (2) is equivalent to maximizing (with respect to $\lambda$ ) the sensitivity $\chi_{T}=\frac{\partial \varphi(\lambda, T)}{\partial T}$, with $f(\lambda)$ assumed to be constant. Indeed:

$$
\chi_{T}=\frac{\partial \varphi(\lambda, T)}{\partial T}=\frac{C_{2}}{\lambda T^{2}}\left(1-\exp \left(\frac{-C_{2}}{\lambda T}\right)\right)^{-1} \varphi(\lambda, T) \underset{\substack{\text { Wien's } \\ \text { approximation }}}{\simeq} \frac{C_{2}}{\lambda T^{2}} \varphi(\lambda, T)
$$

Thus, using the Wien's approximation, maximizing (5) with respect to $\lambda$, leads to (cf. figure 3 for an illustration):

$$
\frac{\partial^{2} \varphi(\lambda, T)}{\partial \lambda \partial T}=0 \Leftrightarrow \lambda_{\text {opt }}^{\text {Wien }} T=\frac{C_{2}}{6} \approx 2400 \mu m K=C_{M W}^{R}
$$




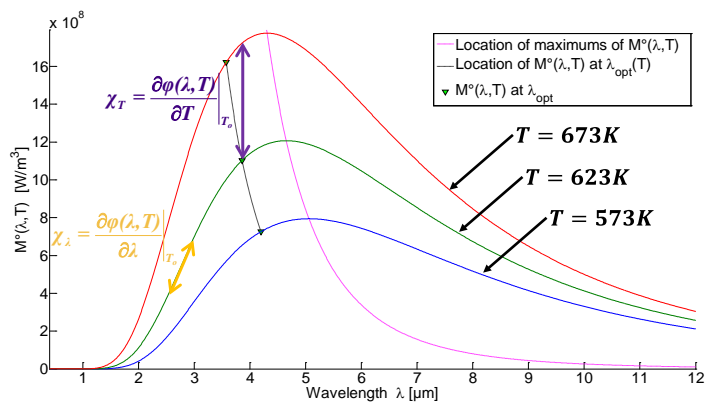

Fig. 3. Graphical illustration of the sensitivities.

Note that, the optimal wavelengths are slightly shifted to the left of the maxima of the Planck's curves (figure 3) and can be interpreted as a compromise between sensitivity and signal over noise ratio (cf. section 4).

\section{The multi-spectral method}

The principle of multi-spectral method is based on the use of multiple wavelengths to obtain the value of different physical quantities. Here, we focus our attention on the temperature and on the coefficients involved in modeling the variation of the emissivity of an oxidized cast iron sample. Among the many existing methods, two particular methods using either direct radiative heat fluxes or their ratios will be briefly presented from a theoretical point of view. Numerical results of estimation will allow us to choose the most adapted method for temperature measurement.

\subsection{Principle of Multi-spectral method based on flux ratio and Wien's approximation}

The principle of the measurement method (called TNL.TXY) is the following: Let $\left(\lambda_{i}, \lambda_{j}\right)_{i<j \leq N_{f}}$ be couples of two wavelengths at which you want to perform our measurements ( $N_{f}$ denotes the number of different wavelengths, in our case, $\left.N_{f}=4\right)$. In practice, these wavelengths correspond to monochromatic filters wavelengths. For each pair of wavelengths, one can calculate the temperature (7) using the flux ratio expressed from the Wien approximation (1) and a model of order 2 (around the mean wavelength $\lambda_{m}$ ) for describing the global spectral transfer function $f(\lambda)$ of the overall system (including the emissivity variations) $[6,9]$.

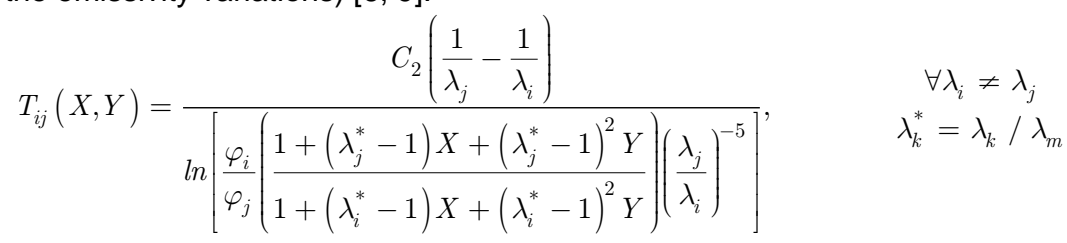

With: $f(\lambda)=f\left(\lambda_{m}\right)+\left(\lambda-\lambda_{m}\right) f^{\prime}\left(\lambda_{m}\right)+\frac{\left(\lambda-\lambda_{m}\right)^{2}}{2} f^{\prime \prime}\left(\lambda_{m}\right), X=\lambda_{m} f^{\prime}\left(\lambda_{m}\right) / f\left(\lambda_{m}\right), Y=\lambda_{m}^{2} f^{\prime \prime}\left(\lambda_{m}\right) / 2 f\left(\lambda_{m}\right)$

The objective is to find the values of $(T, X, Y)$ that minimize the following cost function:

$$
S(T, X, Y)=\sum_{\substack{i=1 \\ j=i+1}}^{N_{f}-1}\left(T-T_{i j}(X, Y)\right)^{2}=\left(T-T_{12}(X, Y)\right)^{2}+\left(T-T_{23}(X, Y)\right)^{2}+\left(T-T_{34}(X, Y)\right)^{2}
$$

\subsection{Principle of Multi-spectral method based on flux}

The previous method is based on the Wien approximation. The estimation model is therefore biased as this will be shown in the numerical validation section. This bias can be significant depending on experimental conditions as for instance the wavelengths working range. That's the reason why, we present here an unbiased model (called TNL.Tabc) based on the estimation of fluxes (left equality of (9)). This model consider a second-order polynomial model for modeling the overall spectral transfer function through three unknown parameters $(a, b, c)$ to estimate. In $(10), \varphi_{i}^{\exp }$ represents the flux measured at the experimental wavelength $\lambda_{i}$.

$$
\varphi_{i}(T, a, b, c)=\underbrace{\left(a+b \lambda_{i}+c \lambda_{i}^{2}\right)}_{=f\left(\lambda_{i}\right)} \frac{C_{1} \lambda_{i}^{-5}}{\exp \left(\frac{C_{2}}{\lambda_{i} T}\right)-1} \underset{\begin{array}{c}
\text { Wien's } \\
\text { approximation }
\end{array}}{\left(a+b \lambda_{i}+c \lambda_{i}^{2}\right)} C_{=f} \lambda^{-5} \exp \left(\frac{-C_{2}}{\lambda T}\right) \quad \forall i \in \llbracket 1 ; \lambda_{f}) \rrbracket
$$


The objective is to find the values of $(T, a, b, c)$ that minimize the following cost function:

$$
J(T, a, b, c)=\sum_{i=1}^{N_{f}}\left(\varphi_{i}^{\exp }-\varphi_{i}(T, a, b, c)\right)^{2}=\left(\varphi_{1}^{\exp }-\varphi_{1}(T, a, b, c)\right)^{2}+\ldots \ldots \ldots . .+\left(\varphi_{4}^{\exp }-\varphi_{4}(T, a, b, c)\right)^{2}
$$

As the method used for temperature estimation is based on the minimization of a functional through an Ordinary Least Squares method (OLS), the idea we propose in this work is to select optimal wavelengths by minimizing the standard deviation of the estimated temperature. In the OLS method, the statistical properties of the parameters (10) are given by the variance-covariance matrix, from which the standard deviations $\sigma_{\beta_{i}}$ of estimated parameters and particularly of the temperature $\sigma_{T}$ can be determined. The model (9) being non-linear, we will use the approximate expression of the variance-covariance matrix of the Ordinary Least Square method, which is given for a parameter vector $\boldsymbol{\beta}=(T, a, b, c)$ , under assumptions of an additive noise, non-correlated, identically distributed (zero mean and constant variance), by:

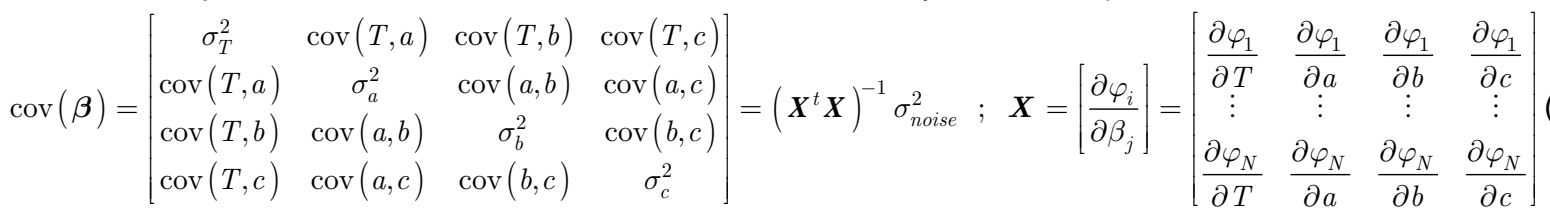

\section{Optimal wavelength selection for temperature measurement}

In this section, firstly we establish analogous Wien's laws to determine the optimal wavelengths for mono-spectral and bi-spectral measurements, then, a general method for multi-spectral measurements is presented. Note that, although this method is presented under assumption of a constant standard deviation of the flux for simplification, it can be applied for a variable standard deviation.

\subsection{Analogous Wien's law for optimal wavelengths selection : mono-spectral and bi-spectral methods}

First, note that for the mono-spectral measurements the minimization of the relative error of the temperature can be linked to the minimization of the standard deviation of the temperature. Indeed, for mono-spectral measurements (one wavelength and one unknown parameter "T" (assumed constant and known emissivity)) from (11) and (6), we have:

$$
\min _{\lambda_{1}}\left(\sigma_{T}\left(\lambda_{1}\right)\right) \Leftrightarrow \min _{\lambda_{1}}\left(\left(\frac{\partial \varphi_{1}}{\partial T}\right)^{-1} \sigma_{\text {noise }}\right) \Rightarrow \frac{\partial^{2} \varphi(\lambda, T)}{\partial \lambda \partial T}=0 \Rightarrow \lambda_{\text {opt }}^{\text {Wien }} T=\frac{C_{2}}{6} \approx 2400 \mu m . K=C_{M}^{W}
$$

Thus, we see from (3), (6) and (12), that there is equivalence between minimizing the relative error on the temperature, maximize the flux sensitivity to the temperature, or minimize the standard deviation of the temperature. This last observation will allow us to generalize the method for determining the optimal wavelengths for bi-spectral and multispectral measurements.

For bi-spectral measurements through Wien's approximation but without flux ratio (i.e. two wavelengths and two unknown parameters " $\{\mathrm{T}, \mathrm{a}\}$ " in (9) and (11) but with Wien's approximation), it is possible to show analytically that the optimal wavelengths which minimize the standard deviation of the temperature (11), are defined by two laws (one for each wavelength) analogous to the Wien's law, such as:

$$
\min _{\lambda_{1}, \lambda_{2}}\left(\sigma_{T}\left(\lambda_{1}, \lambda_{2}\right)\right) \Rightarrow\left\{\begin{array}{l}
\lambda_{o p t 1}^{b i-\text { Wien }} T=C_{B W}^{R 1} \\
\lambda_{\text {opt } 2}^{\text {bi-Wien }} T=C_{B W}^{R 2}
\end{array}\right.
$$

As in the case of mono-spectral measurements, it seems legitimate to extend these laws for fluxes defined by Planck's law. The equations to minimize being once again non-linear, then the approach will be similar as for (4), we will seek numerically the set of pairs of wavelengths $\left(\lambda_{1, i}^{\text {opt }}, \lambda_{2, i}^{\text {opt }}\right)$ that minimize the standard deviation $\sigma_{T_{i}}\left(\lambda_{1}, \lambda_{2}\right)$ of the temperature (calculated from (11) with sensitivities $\boldsymbol{X}$ evaluated at $\boldsymbol{\beta}_{0}=\left(T_{i}, 1\right)$ ), then we will determine the law constants $\left(\hat{C}_{B P}^{R 1}, \hat{C}_{B P}^{R 2}\right)$ using a least squares method, such that:

$$
\left(\hat{C}_{B P}^{R 1}, \hat{C}_{B P}^{R 2}\right)=\arg \min _{\hat{C}_{B P}^{R 1}, \hat{C}_{B P}^{R 2}} \sum_{i}\left(\left(\lambda_{1, i}^{\text {opt }}-\frac{\hat{C}_{B P}^{R 1}}{T_{i}}\right)^{2}+\left(\lambda_{2, i}^{\text {opt }}-\frac{\hat{C}_{B P}^{R 2}}{T_{i}}\right)^{2}\right) \Rightarrow\left\{\begin{array}{l}
\lambda_{\text {opt1 }}^{\text {bi-Planck }} T=C_{B P}^{R 1} \approx 1830.8 \mu m . K \\
\lambda_{\text {opt } 2 \text { blanck }}^{\text {bi-Pla }}=C_{B P}^{R 2} \approx 4465.8 \mu m . K
\end{array}\right.
$$

A graphical representation of wavelengths minimizing standard deviation of the temperature (obtained numerically and for the Planck's law) and those obtained with the laws (14) is given figure 4.

The residues are not shown, but as in the mono-spectral case, the maximum error is of about $10^{-4} \mu \mathrm{m}$. An illustration of optimal wavelengths for mono-spectral and bi-spectral measurements is shown in figure 5. 


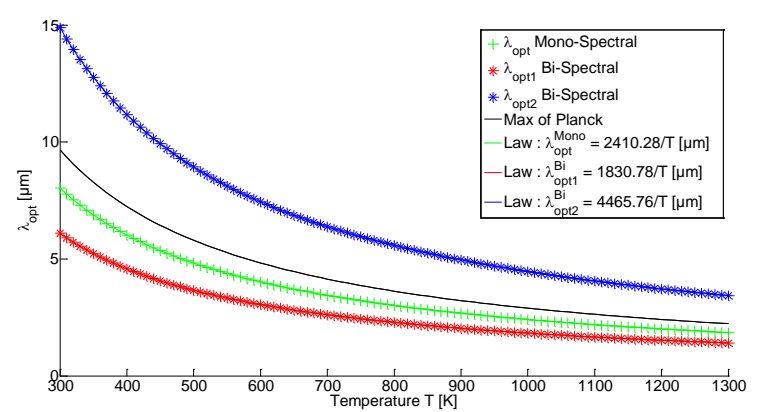

Fig. 4. Optimal wavelengths for bi-spectral measurements. Comparisons between wavelengths obtained numerically and from the laws (14) : $\lambda_{o p t, k}^{\text {bi-Planck }}=\hat{C}_{B P}^{R, k} / T$.

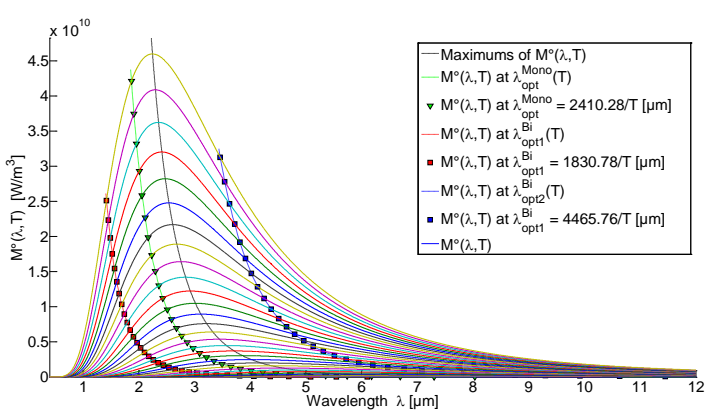

Fig. 5. Illustration on Planck's curves of optimal wavelengths for mono-spectral and bi-spectral measurements.

Note that the optimal wavelengths for bi-spectral measurements satisfy:

$$
\Delta \lambda_{\text {opt }}^{\text {bi-Planck }}=\Delta \lambda_{\text {opt } 2}^{\text {bi-Planck }}-\Delta \lambda_{\text {opt } 1}^{\text {bi-Planck }}=1.9 \underbrace{\frac{\left(\lambda_{\text {opt } 2}^{\text {bi-Planck }}\right)^{2} T}{C_{2}}}_{\Delta \lambda_{\min }}=1.9 \Delta \lambda_{\min }
$$

Where, by differentiating the equation (7) and assimilating the differential terms of errors, we can show [6,9] that $\Delta \lambda_{\min }=\left.\frac{\lambda_{j}^{2} T}{C_{2}}\right|_{\lambda_{j}>\lambda_{i}}$ is the minimum distance between two wavelengths to avoid amplification of measurement errors on the temperature.

\subsection{Optimal wavelengths selection for multi-spectral measurements}

In this section, the methodology used to determine numerically the optimal wavelengths for bi-spectral measurements will be extended to measurements with more than two wavelengths (i.e. for multi-spectral measurements with as many wavelengths as parameters to estimate). Given the criterion $\Delta \lambda_{\min }$ of minimum distance between two wavelengths, for multi-spectral measurements we will use a maximum of four wavelengths $\left(N_{f}=4\right)$ to remain in the spectral range $[1.5 \mu \mathrm{m} ; 5.5 \mu \mathrm{m}]$ of the measuring apparatus (infrared camera). For the same reason, for an emissivity model of order less than two, we choose as many wavelengths as unknown parameters to estimate. The determination of the optimal wavelengths with or without constraints can be done using a global optimization algorithm such as "Trust-Region". Choosing as nominal parameters vector $\boldsymbol{\beta}_{0}=(623 ; 1 ; 1 ; 1)$, for an unconstrained estimation the minimization calculus of the standard deviation of the temperature gives for $\sigma_{\text {noise }} \approx 8.9697 .10^{4} \mathrm{~W}$ (about $7.43 .10^{-3} \%$ of the Planck's law maximum, and equivalent to the value of the experimental noise):

- For $\boldsymbol{\beta}=(T, a, b, c)$, we find: $\boldsymbol{\lambda}_{\text {opt_global }}=\{2.53 ; 4.70 ; 8.87 ; 26.18\} \mu m$ and $\sigma_{T} \approx 0.07 \mathrm{~K}$

- $\quad$ For $\boldsymbol{\beta}=(T, a, b)$, we find: $\boldsymbol{\lambda}_{\text {opt_global }}=\{2.67 ; 5.24 ; 12.57\} \mu \mathrm{m}$ and $\sigma_{T} \approx 0.05 \mathrm{~K}$

- $\quad$ For $\boldsymbol{\beta}=(T, a)$, we find: $\boldsymbol{\lambda}_{\text {opt_global }}=\{2.94 ; 7.17\} \mu m$ and $\sigma_{T} \approx 0.02 \mathrm{~K}$

Although this set of values are the best in terms of minimization of the standard deviation of the temperature, performing measurements at these different wavelengths is difficult in practice because a detector with a so wide spectral range does not exist. For this reason, we decide to choose our wavelengths only in the spectral range of the detector $[1.5 \mu \mathrm{m} ; 5.5 \mu \mathrm{m}]$. If we perform the global optimization with this constraint, the results obtained are:

- $\quad$ For $\boldsymbol{\beta}=(T, a, b, c)$, we find: $\boldsymbol{\lambda}_{\text {opt_cam_spec-range }}=\{2.14 ; 3.39 ; 4.76 ; 5.50\} \mu \mathrm{m}$ and $\sigma_{T} \approx 0.32 \mathrm{~K}$

- $\quad$ For $\boldsymbol{\beta}=(T, a, b)$, we find: $\boldsymbol{\lambda}_{\text {opt_cam_spec-range }}=\{2.43 ; 4.21 ; 5.50\} \mu m$ and $\sigma_{T} \approx 0.09 \mathrm{~K}$

- For $\boldsymbol{\beta}=(T, a)$, we find: $\boldsymbol{\lambda}_{\text {opt_cam_spec-range }}=\{2.93 ; 5.50\} \mu m$ and $\sigma_{T} \approx 0.03 \mathrm{~K}$

Note that in the global estimation with constraints, the last wavelength is always the upper bound, which means that the best wavelength is probably out of the interval.

Experimentally, we have chosen $\boldsymbol{\lambda}_{\exp }=\{2 ; 2.35 ; 2.85 ; 4\} \mu \mathrm{m}$ as wavelengths filters. With these wavelengths, the theoretical standard deviation calculated for $\boldsymbol{\beta}=(T, a, b, c)$ and $\boldsymbol{\beta}_{0}=(623 ; 1 ; 1 ; 1)$, is $\sigma_{T} \approx 2.55 \mathrm{~K}$. 


\section{Numerical validation of models for temperature measurement in the infrared wavelength range}

To validate the model, we simulated no noised (cf. Table 1) and thousand noised fluxes (cf. Table 2) through the Monte-Carlo method (normal noise exhibiting the same level as standard deviation measured on the experimental thermographic images under the same conditions) from four different variations of emissivity (cf. figure 6): constant, linear, order 2 and Drude $\left(\varepsilon_{\lambda} \propto \lambda^{-1 / 2}\right)$. We took care that the variations of order 2 and Drude are significant on the IR spectral range of the study (and in agreement with the experimental behavior of different materials). The tables below show the results for the four filters chosen experimentally. We called $\mathrm{Tm}$, the average temperature of $T_{i j}$ (corresponding to bichromatic measurements) obtained by assuming a constant emissivity ( $X=Y=0$ in (7), or $b=c=0$ in (9)); the notation TNL means that the Temperature is obtained from an "NonLinear least squares" estimation (8) and (10) using the regularized algorithm of "Levenberg-Marquardt". TNL.TXY (respectively TNL.TX) means that we use (8) with $N_{f}=4\left(\right.$ resp. $\left.N_{f}=3\right)$ with the unknown parameters $(T, X, Y)$ (resp. $(T, X))$. Note that when $N_{f}=3$, we take the three shortest wavelengths among the four used to carry out the tables, because it can be shown that the results are better for the shortest wavelengths $[5,6,9]$. Similarly, TNL.Tabc (respectively TNL.Tab), means that we use (10) and the unknown parameters are $(T, a, b, c)($ resp. $(T, a, b))$. For TNL.Tab, we will also take the three shorter wavelengths.

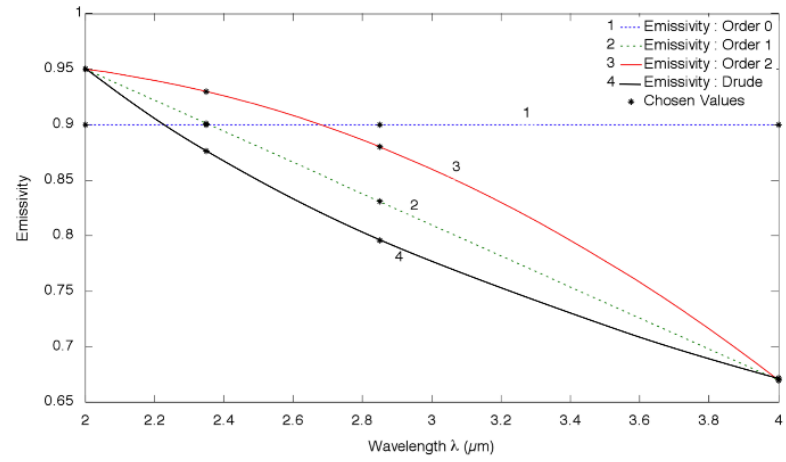

Fig. 6. Emissivity variation used for numerical validation

\subsection{Analysis of Table 1: Simulations without noise}

Table 1. Monte-Carlo simulations for estimating the temperature (without noise)

\begin{tabular}{|c|c|c|c|c|c|c|c|}
\hline \multicolumn{8}{|c|}{$\begin{array}{l}\text { AVERAGE TEMPERATURE OF } 1000 \text { ESTIMATES : lambda }[\mathrm{m}]=\{2 \mathrm{e}-6 ; 2.35 \mathrm{e}-6 ; 2.85 \mathrm{e}-6 ; 4 \mathrm{e}-6\} \\
\text { Texp }=623 \mathrm{~K} ; \text { Radiance law: Planck }\end{array}$} \\
\hline Noise & Emissivity & Model & $\mathrm{T}[\mathrm{K}]$ & Absolute Error $[\mathrm{K}]$ & Relative Error [\%] & Sigma $[\mathrm{K}]$ & Sigma [\%] \\
\hline \multirow{20}{*}{ 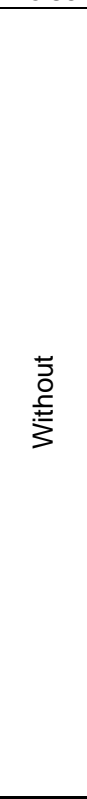 } & \multirow{5}{*}{ Constant } & TNL.TXY (4bands) & 622,47 & 0,53 & 0,09 & - & - \\
\hline & & TNL.TX (3bands) & 623,16 & 0,16 & 0,03 & - & - \\
\hline & & $\mathrm{Tm}$ & 622,87 & 0,13 & 0,02 & - & - \\
\hline & & TNL.Tabc (4bands) & 623,00 & 0,00 & 0,00 & - & - \\
\hline & & TNL.Tab (3bands) & 623,00 & 0,00 & 0,00 & - & - \\
\hline & \multirow{5}{*}{ Linear } & TNL.TXY (4bands) & 622,78 & 0,22 & 0,04 & - & - \\
\hline & & TNL.TX (3bands) & 623,10 & 0,10 & 0,02 & - & - \\
\hline & & $\mathrm{Tm}$ & 651,21 & 28,21 & 4,53 & - & - \\
\hline & & TNL.Tabc (4bands) & 623,00 & 0,00 & 0,00 & - & - \\
\hline & & TNL.Tab (3bands) & 623,00 & 0,00 & 0,00 & - & - \\
\hline & \multirow{5}{*}{ Order 2} & TNL.TXY (4bands) & 622,72 & 0,28 & 0,05 & - & - \\
\hline & & TNL.TX (3bands) & 608,59 & 14,41 & 2,31 & - & - \\
\hline & & $\mathrm{Tm}$ & 643,53 & 20,53 & 3,30 & - & - \\
\hline & & TNL.Tabc (4bands) & 623,00 & 0,00 & 0,00 & - & - \\
\hline & & TNL.Tab (3bands) & 608,52 & 14,48 & 2,32 & - & - \\
\hline & \multirow{5}{*}{ Drude } & TNL.TXY (4bands) & 636,58 & 13,58 & 2,18 & - & - \\
\hline & & TNL.TX (3bands) & 641,17 & 18,17 & 2,92 & - & - \\
\hline & & $\mathrm{Tm}$ & 657,58 & 34,58 & 5,55 & - & - \\
\hline & & TNL.Tabc (4bands) & 636,91 & 13,91 & 2,23 & - & - \\
\hline & & TNL.Tab (3bands) & 641,01 & 18,01 & 2,89 & - & - \\
\hline
\end{tabular}

The non-null estimation uncertainties obtained with TNL.TXY, TNL.TX and Tm for a constant emissivity shows the bias on parameters due to the using of a model based on the Wien approximation (biased model). In particular, the value of this bias is $T m=0.13 \mathrm{~K}$. However, we note that the estimation TNL.TXY (4 bands) gives good results until an order 2 on emissivity variation by correcting partially this bias. The high uncertainties obtained in the case of a Drude 
variation is probably due to the cumulative effect of the biased model used for modeling the emissivity (order 2) and of the Wien / Planck approximation. Compared with the results given by TNL.TX (3 bands), TNL.TXY results are better until a bias on emissivity model appears (order 2 and Drude). As models TNL.Tabc and TNL.Tab do not use Wien's approximation, no bias appears on estimated temperature except for emissivity variation of order 2 for TNL.Tab, and Drude for the models TNL.Tabc and TNL.Tab.

\subsection{Analysis of Table 2: Simulations with noise}

Table 2. Monte Carlo simulations for estimating the temperature (with noise)

\begin{tabular}{|c|c|c|c|c|c|c|c|}
\hline \multirow{2}{*}{\multicolumn{8}{|c|}{ 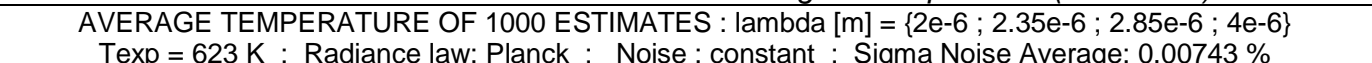 }} \\
\hline & & & & & & & \\
\hline Noise & Emissivity & Model & $\mathrm{T}[\mathrm{K}]$ & Absolute Error [K] & Relative Error [\%] & Sigma $[\mathrm{K}]$ & Sigma [\%] \\
\hline \multirow{20}{*}{$\stackrel{5}{3}$} & \multirow{5}{*}{ Constant } & TNL.TXY (4bands) & 622,48 & 0,52 & 0,08 & 1,11 & 0,18 \\
\hline & & TNL.TX (3bands) & 623,16 & 0,16 & 0,03 & 0,52 & 0,08 \\
\hline & & $\mathrm{Tm}$ & 622,87 & 0,13 & 0,02 & 0,08 & 0,01 \\
\hline & & TNL.Tabc (4bands) & 623,02 & 0,02 & 0,00 & 1,13 & 0,18 \\
\hline & & TNL.Tab (3bands) & 623,00 & 0,00 & 0,00 & 0,52 & 0,08 \\
\hline & \multirow{5}{*}{ Linear } & TNL.TXY (4bands) & 622,78 & 0,22 & 0,03 & 0,69 & 0,11 \\
\hline & & TNL.TX (3bands) & 623,10 & 0,10 & 0,02 & 0,32 & 0,05 \\
\hline & & Tm & 651,21 & 28,21 & 4,53 & 0,07 & 0,01 \\
\hline & & TNL.Tabc (4bands) & 623,01 & 0,01 & 0,00 & 0,70 & 0,11 \\
\hline & & TNL.Tab (3bands) & 623,00 & 0,00 & 0,00 & 0,32 & 0,05 \\
\hline & \multirow{5}{*}{ Order 2} & TNL.TXY (4bands) & 622,73 & 0,27 & 0,04 & 1,04 & 0,17 \\
\hline & & TNL.TX (3bands) & 608,59 & 14,41 & 2,31 & 0,30 & 0,05 \\
\hline & & $\mathrm{Tm}$ & 643,53 & 20,53 & 3,30 & 0,07 & 0,01 \\
\hline & & TNL.Tabc (4bands) & 623,02 & 0,02 & 0,00 & 1,05 & 0,17 \\
\hline & & TNL.Tab (3bands) & 608,52 & 14,48 & 2,32 & 0,30 & 0,05 \\
\hline & \multirow{5}{*}{ Drude } & TNL.TXY (4bands) & 636,59 & 13,59 & 2,18 & 0,73 & 0,12 \\
\hline & & TNL.TX (3bands) & 641,17 & 18,17 & 2,92 & 0,38 & 0,06 \\
\hline & & $\mathrm{Tm}$ & 657,58 & 34,58 & 5,55 & 0,07 & 0,01 \\
\hline & & TNL.Tabc (4bands) & 636,91 & 13,91 & 2,23 & 0,74 & 0,12 \\
\hline & & TNL.Tab (3bands) & 641,01 & 18,01 & 2,89 & 0,38 & 0,06 \\
\hline
\end{tabular}

Note that the noise is additive to the flux, but not to the temperature according to (7). The noise will thus lead to a bias on estimated parameters with TNL.TXY, TNL.TX and Tm models. However, we can see that it is impossible by the methods TNL.TXY and TNL.Tabc to accurately estimate the temperature because the problem seems to be ill-posed. This observation is confirmed by the best results given by TNL.TX and TNL.Tab in the case of emissivities ranging up to order 2 , which shows that it is possible to regularize the problem by reducing the number of parameters. Nevertheless, it is important to note that the standard deviations of the estimations are significant, suggesting that it will be necessary to have a lot of points or to use larger integration times if we want to increase the measurement accuracy. From these results, we will choose the TNL.Tabc model as estimation model for the experiments.

\section{Experimental results}

\subsection{Description of the experimental bench}

The diagram of the facility is shown in figure 7. An oxidized cast iron sample on which "FT 25" is engraved (its surface being varied, so it is for the emissivity) is placed in a tube furnace at a temperature of 623K controlled by a PID with a great stability (no oscillations in temperature recording due to furnace regulation). The temperature of the sample is obtained using a thermocouple placed on its rear face. The spatial radiative flux emitted by the sample is measured through a high sensitive Broad-Band InSb infrared matrix camera working in the spectral range [1.5 $\mu \mathrm{m}$; $5.5 \mu \mathrm{m}]$. Four monochromatic filters: $\boldsymbol{\lambda}_{\exp }=\{2 ; 2.35 ; 2.85 ; 4\} \mu \mathrm{m}$ are mounted in the filters wheel of this camera in order to measure the emitting flux coming from the sample at four different wavelengths. The signal is digitized through a 14 bits Analog/Digital card. Each pixel is associated to a Digital Level (DL) corresponding to the spectral radiance of a surface area of the sample. The camera has previously been calibrated in the temperature range [573K-673K] using a 4"x4" extended area blackbody. 


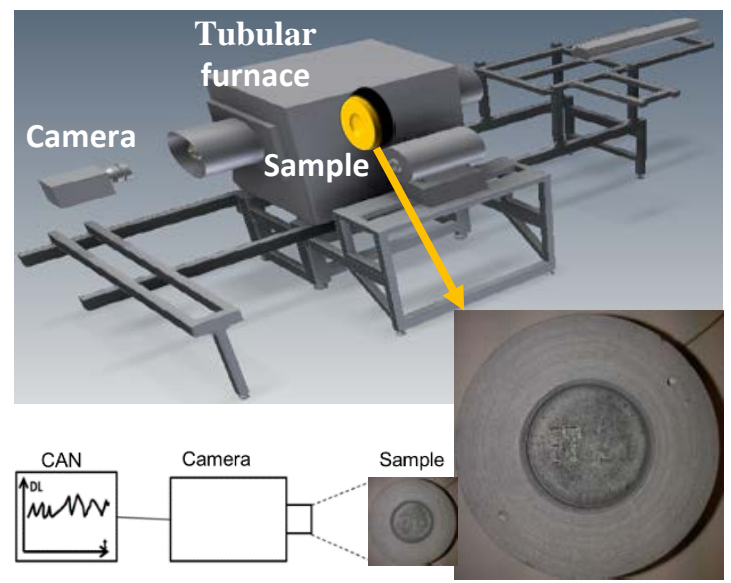

Fig. 7. Facility for IR measurements

\subsection{Measurement methodology, data processing and results}

Using a tubular furnace, the sample is heated at three different temperatures levels $\{\mathrm{T} 1=573 \mathrm{~K}, \mathrm{~T} 2=623 \mathrm{~K}, \mathrm{~T} 3=$ $673 \mathrm{~K}\}$. For each temperature, a recording of 1000 images for each filter is performed, taking care before each acquisition that the thermal equilibrium was reached. To get free of the reflection through the non-blackbody sample that is not negligible at this level of temperature due to presence of the hot furnace walls in this vicinity, we use the average image made with 4 filters at $573 \mathrm{~K}$ and $673 \mathrm{~K}$ to correct the existing offset between our measurements at these two temperatures and the flux that a blackbody at these same temperatures would emit. Calling $M_{\lambda_{i}}^{\exp }\left(T_{j}\right)$ the experimental heat flux measured at the wavelengths $\lambda_{i}$ and at the temperature $T_{j}$, and $K_{i}$ a variable to correct the offset between the measured flux and the blackbody flux, we have a set of 8 equations with 8 unknowns (the four couples $\left(\varepsilon_{i} ; K_{i}\right)$ ) to solve. The system is as follows:

$$
M_{\lambda_{i}}^{\exp }\left(T_{j}\right)=\varepsilon_{i} M_{\lambda_{i}}^{o}+K_{i} \quad, \forall i \in \llbracket 1 ; 4 \rrbracket \text { and } \forall j \in\{1 ; 3\}
$$

The 8 unknowns $\left(\varepsilon_{i} ; K_{i}\right)$ are estimated by a regularized ordinary least square method (Levenberg-Marquardt).

Using the $K_{i}$ (assuming the reflected part of the heat flux as constant in the temperature range 573K-673K), our experimental flux can be corrected to get free from the reflection. Finally, the experimental flux is corrected for each pixel through the following relation:

$$
\varphi_{\lambda_{i}}^{\exp }\left(T_{j}\right)=M_{\lambda_{i}}^{\exp }\left(T_{j}\right)-K_{i} \quad, \forall i \in \llbracket 1 ; 4 \rrbracket \text { and } \forall j \in \llbracket 1 ; 3 \rrbracket
$$

The aim is now to estimate using adjusted flux emitted by each pixel, the temperature field of the sample when the furnace is at $T_{2}=623 \mathrm{~K}$. For this, we will find for each pixel the value of temperature $T$ that minimizes the cost function (10). Figures 8-11 show the averaged (1000 Images) thermographic images recorded by the camera through the four monochromatic filters $\boldsymbol{\lambda}_{\exp }=\{2 ; 2.35 ; 2.85 ; 4\} \mu \mathrm{m}$. The inscription "FT25" is indistinguishable at $2 \mu \mathrm{m}$ but appears more and more clearly up to $4 \mu \mathrm{m}$. The result of the temperature estimation by inversion of Planck's law (assuming unit emissivity) is given in Figure 12 for the wavelength at $4 \mu \mathrm{m}$, corresponding to the experimental wavelength closest to the theoretical optimal wavelength defined by (4). As expected, we note that this simple estimation assuming a uniform emissivity does not correct the emissivity field because the pattern "FT25" is still visible on the calculated temperature field. Moreover, the estimation error of temperature is large, experimental temperature is about $623 \mathrm{~K}$ while the estimated temperature is about $588 \mathrm{~K}$ (approximately $6 \%$ error or $35 \mathrm{~K}$ ) with a standard deviation of about $0.52 \mathrm{~K}$. The figure 13 illustrates the result of the estimated temperature by bi-spectral method (i.e. (7) with $X=Y=0$, or (9) with $b=c=0$ ) with $\lambda_{\exp }=\{2 ; 2.35\} \mu m$. The shortest wavelengths have been chosen because they are closest, which will facilitate the assumption of constant emissivity. This assumption appears to be relatively good, because the estimated temperature is about 516K (approximately $1 \%$ error or $7 \mathrm{~K}$ ) and the standard deviation is about 3.6K. The figure 14 shows the influence of a bias model (no constant emissivity), and thus the advantage of choosing wavelengths as close as possible. Indeed, figure 14 represents the estimated temperature field (about 596K, with standard deviation of $2.3 \mathrm{~K}$ ) given by the mean of the three bi-spectral temperatures $T_{i j}$ : Eq. (7) with $i=1, j \in \llbracket 2 ; 4 \rrbracket$, and $X=Y=0$. We see that the temperature error (about $27 \mathrm{~K}$ or $4 \%$ ) is much greater than in the bi-spectral case because of the excessive distance between the wavelengths, not favoring the assumption of constant emissivity. Figure 15 shows the result of the estimated temperature field (about $625 \mathrm{~K}$ ) given by the TNL.Tabc model (minimization of the functional (10)). We note that the pattern has also (as for the bi-spectral methods) totally disappeared and that the temperature error is about $2 \mathrm{~K}(0.3 \%)$ with a standard deviation of $4 \mathrm{~K}$. Note that, we have also tried the TNL.Tab model (with 3 or 4 wavelengths) but the best results are given by the model TNL.Tabc. 

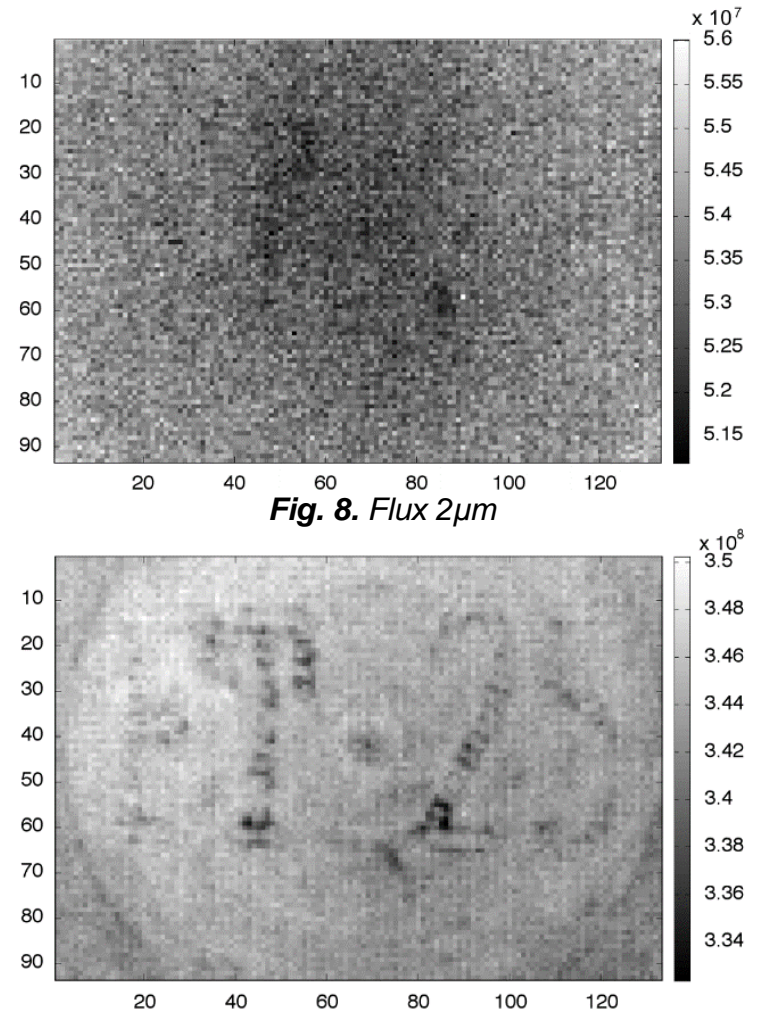

Fig. 10. Flux $2.85 \mu m$

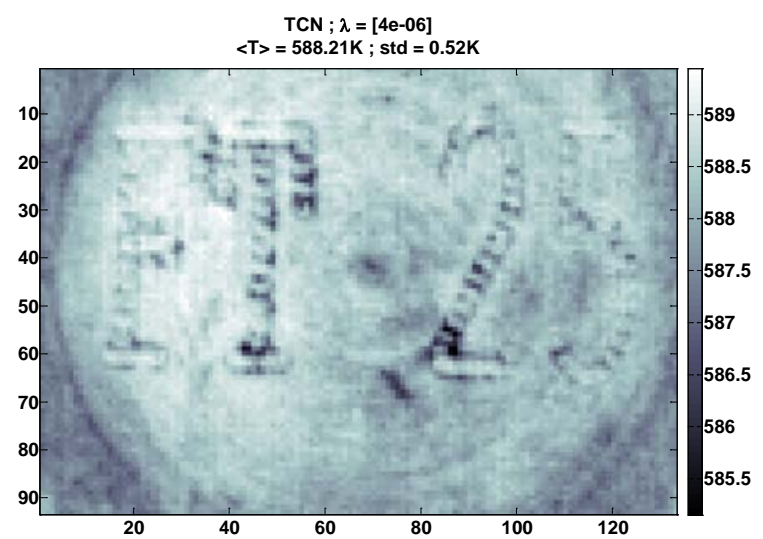

Fig. 12. Estimated temperature: TCN $4 \mu \mathrm{m}$

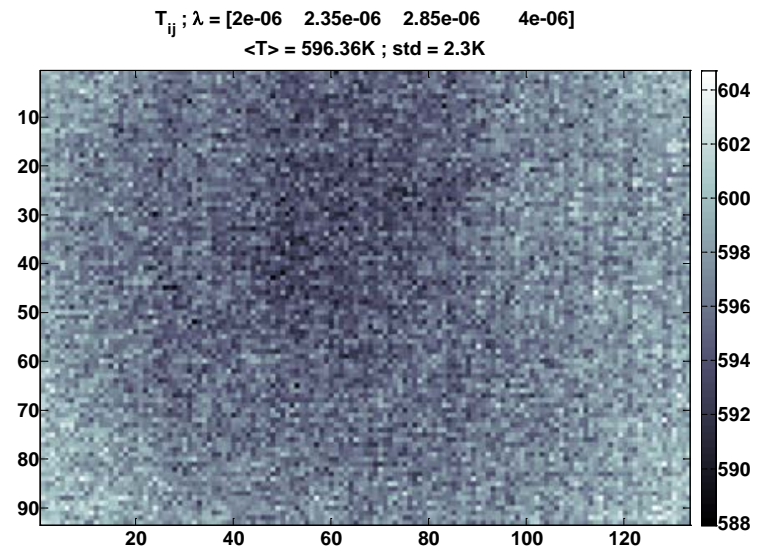

Fig. 14. Estimated temperature: $T_{m}$ (average of $T_{\text {bispectral) }}$
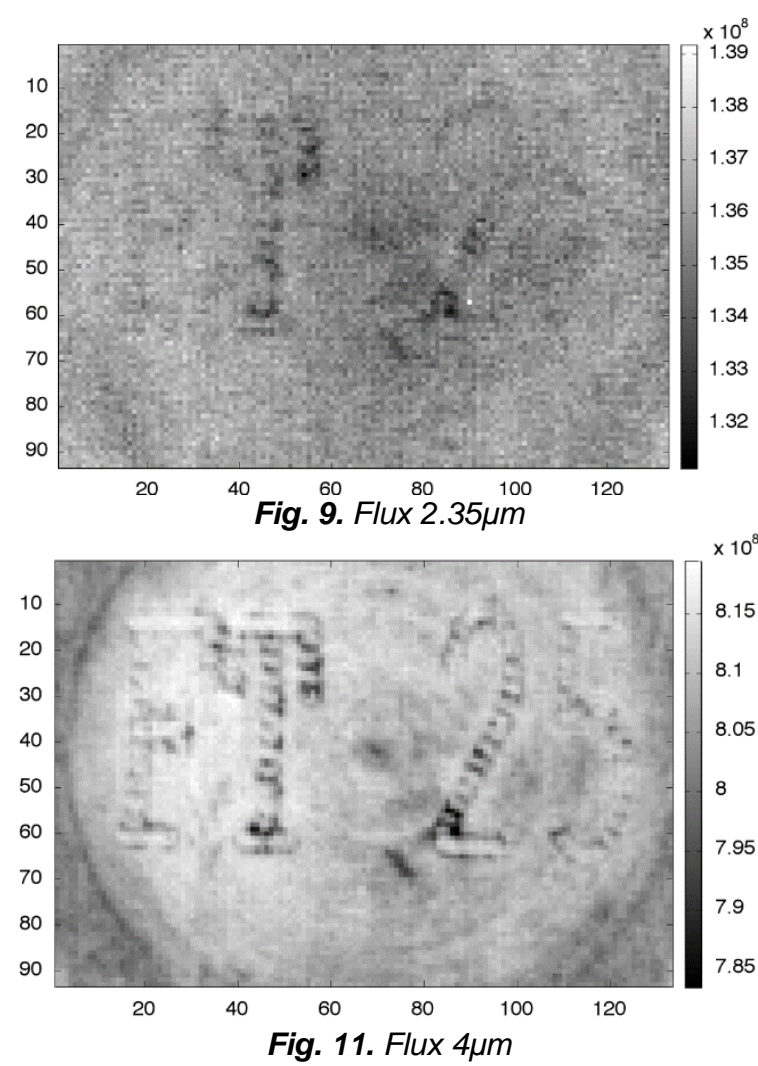

$\mathrm{T}_{\mathrm{ij}} ; \lambda=\left[\begin{array}{ll}2 \mathrm{e}-06 & 2.35 \mathrm{e}-06\end{array}\right]$

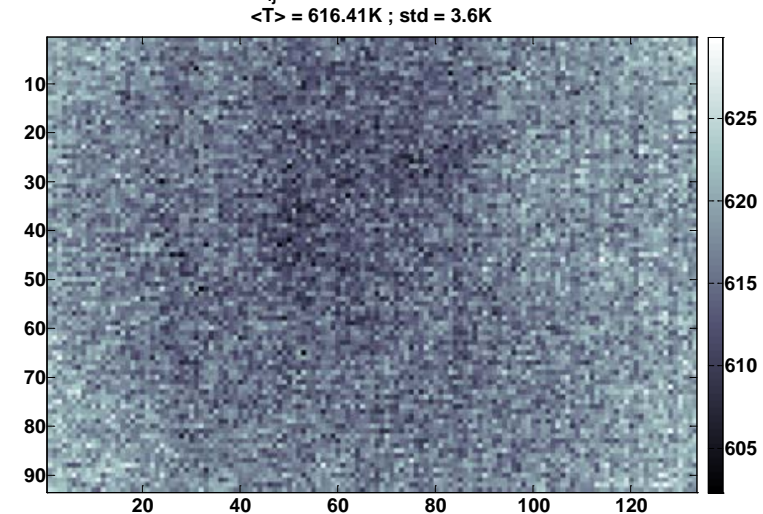

Fig. 13. Estimated temperature: $T_{\text {bispectral }}$

Tabc $; \lambda=\left[\begin{array}{llll}2 \mathrm{e}-06 & 2.35 \mathrm{e}-06 & 2.85 \mathrm{e}-06 & 4 \mathrm{e}-06\end{array}\right]$ $\langle\mathrm{T}\rangle=625.69 \mathrm{~K} ; \mathrm{std}=4 \mathrm{~K}$

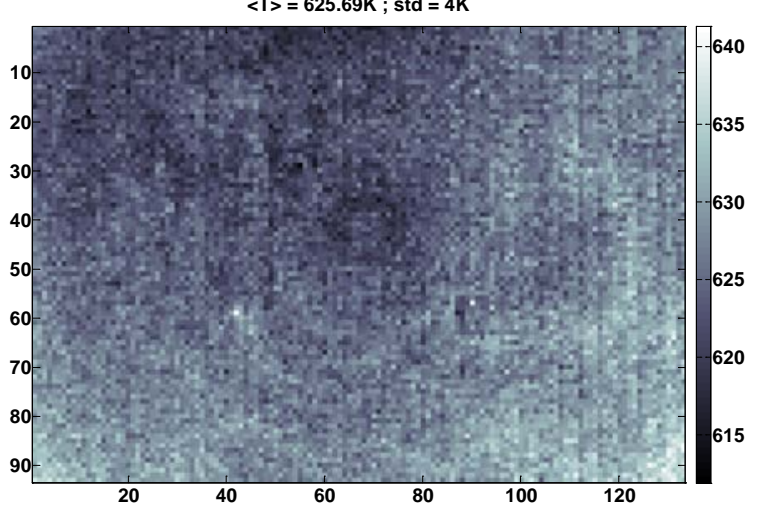

Fig. 15. Estimated temperature: $T_{a b c}$ 


\section{Conclusion}

Firstly, analogous Wien's laws for optimal wavelengths selection in the sense of minimizing the relative error or the standard deviation of the temperature have been presented for mono-spectral and bi-spectral measurements (for fluxes defined from Planck's law). In addition, the relationship between minimizing the relative error of temperature, minimizing the standard deviation of temperature, and maximizing the sensitivity of the flux to the temperature, has been established. Then, a general method based on an ordinary least squares method with or without constraints on the spectral range has been proposed for multi-spectral measurements.

Next, it was shown through Monte-Carlo simulations (table 1 and table 2) that the TNL.TXY model is biased due to on the one hand to the Wien approximation, and on the other hand, due to the non-additive uncertainty on fluxes that lead to a systematic uncertainty on the estimated temperature T. However, the results given by the unbiased model TNL.Tabc (9) (using fluxes without Wien's approximation and without fluxes ratio) and summarized in Table 2 are very satisfactory for emissivity variations of order between 0 and 2 (Drude model is a more difficult case).

To finish, the experimental results obtained using different methods and from the filters available experimentally $\boldsymbol{\lambda}_{\exp }=\{2 ; 2.35 ; 2.85 ; 4\} \mu m$ have been compared. The different models compared are the radiance temperature, the bispectral temperature (with 2 or 4 wavelengths), and the TNL.Tabc model. The results obtained with the TNL.Tabc model are very encouraging with an uncertainty of about $2 \mathrm{~K}(0.3 \%)$ and a standard deviation of $4 \mathrm{~K}$.

To conclude, we have proposed in this work an unbiased multi-spectral method to estimate through an ordinary least squares method the temperature of surfaces exhibiting non-uniform emissivity up to a second order variation. Analogous Wien's laws for optimal wavelengths selection for mono-spectral and bi-spectral methods and a new criteria based on the minimization of the standard deviation of the estimated temperature and has also been proposed. It allowed us to define in an optimal way the different wavelengths to use for the temperature measurement.

\section{REFERENCES}

[1] Souhar Y, Rémy B, Degiovanni A. Thermal Characterization of Anisotropic Materials at High Temperature through Integral Methods and Localized Pulsed Technique. International Journal of Thermophysics. 2013;34:322-340.

[2] Gardner JL, Jones TP, Davies MR. A six wavelength radiation pyrometer. High Temperatures - High Pressures. 1981;13:459-466.

[3] Meriaudeau F. Real time multispectral high temperature measurement: application to control in industry. Image and Vision Computing. 2007;25(7):1124-1133.

[4] Duvault T. Comparison between multiwavelength infrared and visible pyrometry: application to metals. Infrared Physics \& Technology. 2008;51(4):292-299.

[5] Duvault T. Multiwavelength infrared pyrometry: optimization and computer simulations. Infrared Physics \& Technology. 1995;36(7):292-299.

[6] Pierre T, Rémy B, Degiovanni A. Micro-scale temperature measurement by multi-spectral and statistic method in the ultraviolet-visible wavelengths. Journal of Applied Physics. 2008;103-1.

[7] Hervé P, Cedelle J, Negreanu I. Infrared technique for simultaneous determination of temperature and emissivity. Infrared Physics \& Technology. 2012 Jan;55(1):1089-1103.

[8] Krapez J-C. Radiative measurements of temperature. In: Orlande HRB, Fudym O., Maillet D., Cotta RM, editors. Thermal measurements and inverse techniques. Boca Raton: CRC Press, Taylor \& Francis Group, 2011, pp. 185-230.

[9] Rodiet C, Rémy B, Degiovanni A, and Demeurie F. Optimisation of wavelengths selection used for the multispectral temperature measurement by ordinary least squares method of surfaces exhibiting non-uniform emissivity. Quantitative InfraRed Thermography Journal, 10(2):222-236, 2013. 